\title{
A Distributed Set-membership Approach based on Zonotopes for Interconnected Systems
}

\author{
Ye Wang ${ }^{1}$, Teodoro Alamo ${ }^{2}$, Vicenç Puig ${ }^{1}$ and Gabriela Cembrano ${ }^{1,3}$
}

\begin{abstract}
This paper proposes a distributed set-membership approach based on zonotopes for interconnected systems with coupled states and unknown-but-bounded uncertainties (both state disturbances and measurement noise). The objective of the distributed set-membership approach is to find a sequence of distributed zonotopes to bound uncertain states of each subsystem (called agent) instead of making use of a single zonotope to bound all the uncertain states. In the proposed approach, these distributed state bounding zonotopes are only corrected by their own measurement outputs. To predict the state at the next sampling time, each agent sends its own statebounding zonotope to its neighbors. For achieving robust state estimation, we propose a novel optimization problem based on the $P$-radius minimization criterion. Finally, the effectiveness of the proposed approach is provided with a numerical example.
\end{abstract}

\section{INTRODUCTION}

As society develops, an increasing number of large-scale systems, such as cyber-physical systems [1] and critical infrastructures (i.e. water distribution networks [2] and smart grids [3]), are becoming more automatized. Such kind of systems have a large amount of states, inputs and outputs. Considering their complexity and dimension, these largescale systems can be formulated as interconnected systems with coupled states. In the frameworks of diagnosis and optimal control of large-scale systems, a suitable distributed state estimation approach plays a significant role in the development of model-based fault diagnosis strategies [4] and the design of controllers [5], [6]. Revising the literature, different approaches have been investigated for distributed state estimation problems, as e.g. the distributed moving horizon approaches in [7], [8].

Set-membership approaches for robust state estimation have been discussed over the last decade (see, e.g. [9], [10], [11], [12], [13], [14]). The idea behind the set-membership approach is to use customized geometrical sets to bound uncertain states during iterations. The alternative geometrical sets include intervals, ellipsoids, polytopes, zonotopes among others. Due to their low computational complexity, zonotopes, a type of symmetrical sets, are usually chosen; see for instance [10]. However, most of existing approaches

\footnotetext{
${ }^{1}$ Y. Wang, V. Puig and G. Cembrano are with Advanced Control Systems (SAC) Research Group at Institut de Robòtica i Informàtica Industrial (IRI), CSIC-UPC, Universitat Politècnica de Catalunya-BarcelonaTech (UPC), C/. Llorens i Artigas 4-6. 08028 Barcelona, Spain. E-mail: ywang@iri.upc.edu, vicenc.puig@upc.edu, cembranodiri.upc.edu

${ }^{2} \mathrm{~T}$. Alamo is with Departamento de Ingeniería de Sistemas y Automática, Universidad de Sevilla, Avenida de los Descubrimientos, S/N, 41092 Sevilla, Spain. E-mail: talamodus.es

${ }^{3}$ G. Cembrano is also with CETaqua, Water Technology Centre, Ctra d'Esplugues 75, Cornellà de Llobregat, 08940 Barcelona, Spain.
}

only consider the construction of single set for bounding all the uncertain states in a centralized way. For largescale systems, since the system dimension is very high, it would be very difficult to propagate a high-dimensional set at each time step. Naturally, the set-membership approach could be extended to work in a distributed way. We studied a distributed set-membership approach with partial projection in [15], where coupled states are assumed to be measured.

In the literature, a distributed set-membership observer for interconnected multi-rate systems is proposed in [16], where a state observer is constructed using zonotopes and the observer gain is computed by means of the zonotopic Kalman observer approach as discussed in [13]. In this paper, we propose a different approach based on zonotopes [10]. We define a new structure of parameterized distributed state bounding zonotopes, where the consistency between the system model and the independent measurement outputs is established. Furthermore, the parameter matrices for the construction of these distributed zonotopes are determined solving an optimization problem. Here, as an improvement of our previous work [15], coupled states are taken into account.

The main contribution of this paper is to propose a distributed set-membership approach for interconnected systems with coupled states. Unknown-but-bounded state disturbances and measurement noise are considered in the system. Throughout the paper, we bound the uncertainty associated with the state estimation by zonotopes. Firstly, we define the parameterized distributed state bounding zonotope for each interconnected system. For obtaining the robust state estimation results, we then propose an optimization problem to minimize the effect of uncertainties based on the $P$-radius minimization criterion in order to find the parameter of the distributed state bounding zonotope. Finally, some simulation results with a numerical example are shown.

The remainder of this paper is organized as follows. In Section III, the problem statement is addressed. In Section IV, the main results including the structure of distributed state bounding zonotopes and the distributed set-membership state estimation is presented. In Section V, a numerical example is provided to illustrate the proposed distributed setmembership approach. Finally, conclusions are outlined in Section VI.

Notation: Throughout this paper, we use $I_{r}$ to denote an identity matrix with dimension $r$. We denote the Minkowski sum as $\oplus$. For a matrix $A$, we denote $\operatorname{tr}(A)$ and $\operatorname{rank}(A)$ as the trace and the rank of $A$ respectively, $A^{-1}$ and $A^{T}$ as the inverse and the transpose of $A$ respectively, and $A \succ 0$ denotes positive definiteness. For two matrices $A$ and $B$, 
we denote $\operatorname{diag}(A, B)$ as the corresponding block diagonal matrix. For a set of matrices $A_{j}$ with $j \in N$, we denote

$$
\operatorname{cat}_{j \in N}\left\{A_{j}\right\}=\left[A_{j_{1}}, A_{j_{2}}, \ldots, A_{j_{N}}\right],
$$

where $j_{1}<j_{2}<\cdots<j_{N}$ are the (ordered) elements of $N$. Besides, we define the following sets $\mathbb{S}^{n} \quad:=\quad\left\{X \in \mathbb{R}^{n \times n} \mid X=X^{T}\right\}, \quad \mathbb{S}_{\succ 0}^{n} \quad:=$ $\left\{X \in \mathbb{R}^{n \times n} \mid X=X^{T}, X \succ 0\right\} \quad$ and $\quad \mathbb{S}_{\succeq 0}^{n} \succ 0 \quad:=$ $\left\{X \in \mathbb{R}^{n \times n} \mid X=X^{T}, X \succeq 0\right\}$. For a vector $z \in \mathbb{R}^{n}$, we use $\|z\|_{1}$ and $\|z\|_{\infty}$ to denote the 1-norm and the infinity norm of $z$, respectively. Moreover, for a vector $z$, we use $\operatorname{diag}(z)$ to denote a diagonal matrix in which the elements in the diagonal are the components of $z$.

\section{Preliminaries}

In this section, we introduce some necessary definitions and properties related to zonotopes.

Definition 1 (Zonotope): Given a vector $c \in \mathbb{R}^{n}$ and a matrix $H \in \mathbb{R}^{n \times r}$, a zonotope $\mathcal{Z} \subset \mathbb{R}^{n}$ in $n$-dimensional space is defined as

$$
\mathcal{Z}:=\langle c, H\rangle=\left\{c+H z,\|z\|_{\infty} \leq 1\right\},
$$

where $c$ and $H$ are called the center and the segment matrix of the zonotope $\mathcal{Z}$.

With the Minkowski sum $\oplus$, the zonotope in (1) can also be defined by $\mathcal{Z}=c \oplus H \mathbf{B}^{r}$, where $\mathbf{B}^{r}=[-1,+1]^{r} \subset \mathbb{R}^{r}$ is an $r$-dimensional hypercube. Besides, the following linear properties hold:

$$
\begin{aligned}
\left\langle c_{1}, H_{1}\right\rangle \oplus\left\langle c_{2}, H_{2}\right\rangle & =\left\langle c_{1}+c_{2},\left[\begin{array}{ll}
H_{1} & H_{2}
\end{array}\right]\right\rangle, \\
L\langle c, H\rangle & =\langle L c, L H\rangle
\end{aligned}
$$

where $L$ is an arbitrary matrix of appropriate dimension.

In the following definition, a measure of the size of a zonotope $\mathcal{Z}$ is introduced [17].

Definition 2 (P-radius of a zonotope): Given a zonotope $\mathcal{Z}=\langle p, H\rangle \subset \mathbb{R}^{n}$ and a matrix $P \in \mathbb{S}_{\succ 0}^{n}$, the $P$-radius of $\mathcal{Z}$ is defined by

$$
V(\mathcal{Z}):=\max _{\|z\|_{\infty} \leq 1}\|H z\|_{P}^{2}=\max _{\|z\|_{\infty} \leq 1} z^{T} H^{T} P H z .
$$

In the following result, in order to reduce the dimension of a zonotope $\mathcal{Z}$, an over-bounding parallelotope can be obtained.

Lemma 1 ([11]): Given a zonotope $\mathcal{Z}=c \oplus H \mathbf{B}^{r}$, where $H \in \mathbb{R}^{n \times r}, \operatorname{rank}(H)=n$ with $n \leq r$. Consider the singular value decomposition $H=U \Sigma V^{T}$, where $\Sigma=$ $\operatorname{diag}(\sigma)$ and $\sigma=\left[\sigma_{1}, \ldots, \sigma_{n}\right]^{T}$. The zonotope $\mathcal{Z}$ can be over-bounded by the parallelotope $\mathcal{Z}^{p}=\langle c, U D\rangle$, that is, $\mathcal{Z} \subseteq \mathcal{Z}^{p}=c \oplus U D \mathbf{B}^{n}$, where $D$ is a diagonal matrix with the diagonal components $D_{i i}=\left\|\sigma_{i} V_{i}\right\|_{1}$, for $i=1, \ldots, n$ and $V_{i}$ is the $i$-th column of the matrix $V$.

\section{Problem Statement}

Consider the class of discrete-time systems that can be decomposed into $l$ interconnected subsystems (called agents) with coupled dynamics. Each agent is modeled in discretetime as follows:

$$
\begin{aligned}
x_{i}(k+1) & =\sum_{j \in N_{i}} A_{i j} x_{j}(k)+B_{i} u_{i}(k)+w_{i}(k), \\
y_{i}(k) & =C_{i} x_{i}(k)+v_{i}(k),
\end{aligned}
$$

where $x_{i} \in \mathbb{R}^{n_{i}}, u_{i} \in \mathbb{R}^{m_{i}}$ and $y_{i} \in \mathbb{R}^{p_{i}}$ denote the state, the input and the output vectors, $w_{i} \in \mathbb{R}^{m_{w_{i}}}$ and $v_{i} \in \mathbb{R}^{m_{v_{i}}}$ denote the state disturbance and the measurement noise vectors of the $i$-th agent with $i=1, \ldots, l$, respectively. $A_{i i} \in \mathbb{R}^{n_{i} \times n_{i}}, B_{i} \in \mathbb{R}^{n_{i} \times m_{i}}$ and $C_{i} \in \mathbb{R}^{p_{i} \times n_{i}}$. Besides, $N_{i}$ is defined to be the set that includes all the agents related to the agent $i$ ( $i$ also included).

In this paper, $w_{i}(k)$ and $v_{i}(k), \forall k \in \mathbb{N}$ are unknown at each time step. To design an iterative approach, the following assumptions are made.

Assumption 1: The state disturbance and measurement noise vectors $w_{i}(k)$ and $v_{i}(k)$ are unknown but bounded by the centered zonotopes:

$$
w_{i}(k) \in\left\langle 0, D_{w_{i}}\right\rangle, v_{i}(k) \in\left\langle 0, D_{v_{i}}\right\rangle, \forall k \in \mathbb{N},
$$

and for $i=1, \ldots, l$, where $D_{w_{i}} \in \mathbb{R}^{m_{w_{i}} \times m_{w_{i}}}$ and $D_{v_{i}} \in$ $\mathbb{R}^{m_{v_{i}} \times m_{v_{i}}}$.

Assumption 2: The initial state $x_{i}(0)$ is assumed to be bounded by the zonotope $x_{i}(0) \in \mathcal{X}_{i}(0):=\left\langle c_{i}(0), H_{i}(0)\right\rangle$ for $i=1, \ldots, l$.

In this work, the goal is to obtain robust state estimations of uncertain states in presence of unknown state disturbances and measurement noise by finding a sequence of distributed state zonotopes $\mathcal{X}_{i}(k)$ to independently bound the uncertain states $x_{i}(k)$ for $i=1, \ldots, l, \forall k \in \mathbb{N}$. These distributed state zonotopes provide robust state estimation results.

\section{MAIN RESULT}

In this section, we present a distributed set-membership approach for robust state estimation. A parameterized distributed state bounding zonotope is established for each agent considering coupled states. To determine the parameters (correction matrices) of the distributed state bounding zonotopes for robust state estimation, we propose a novel optimization problem based on $P$-radius minimization. Finally, the distributed set-membership state estimation algorithm is presented.

\section{A. Distributed State Bounding Zonotope}

Instead of finding a single state bounding zonotope, we introduce the structure of the parameterized distributed state bounding zonotope $\mathcal{X}_{i}(k)$ for $i=1, \ldots, l$ and $\forall k \in \mathbb{N}$. In this case, the coupled states are considered. Each zonotope $\mathcal{X}_{i}(k)$ is built to be consistent with the individual measurement output $y_{i}(k)$ of each agent. Considering that the initial states $x_{i}(0)$ are assumed to be bounded by state zonotopes, the parameterized distributed state bounding zonotopes are recursively defined in the following proposition.

Proposition 1 (Distributed state bounding zonotope): Given the dynamics of the distributed systems in (4), suppose that Assumption 1 and 2 hold, and that 


$$
\begin{aligned}
c_{i}(k) & =\left(I-\Lambda_{i} C_{i}\right)\left(\sum_{j \in N_{i}} A_{i j} c_{j}(k-1)+B_{i} u_{i}(k-1)\right)+\Lambda_{i} y_{i}(k), \\
H_{i}(k) & =\left[\left(I-\Lambda_{i} C_{i}\right) \operatorname{cat}_{j \in N_{i}}\left\{A_{i j} H_{j}(k-1)\right\}, \quad\left(I-\Lambda_{i} C_{i}\right) D_{w_{i}}, \quad \Lambda_{i} D_{v_{i}}\right] .
\end{aligned}
$$

$x_{i}(k-1) \in \mathcal{X}_{i}(k-1)=\left\langle c_{i}(k-1), H_{i}(k-1)\right\rangle$, $i=1, \ldots, l$. Then, the following inclusion holds for every correction matrix $\Lambda_{i} \in \mathbb{R}^{n_{i} \times p_{i}}$ :

$$
x_{i}(k) \in \mathcal{X}_{i}(k)=\left\langle c_{i}(k), H_{i}(k)\right\rangle, i=1, \ldots, l,
$$

where $c_{i}(k)$ and $H_{i}(k)$ are defined in (7).

Proof: Since $x_{i}(k-1) \in\left\langle c_{i}(k-1), H_{i}(k-1)\right\rangle$ for $i=$ $1, \ldots, l$, by Definition 1 , there exists a vector $\theta_{i}(k-1)$ with $\left\|\theta_{i}(k-1)\right\|_{\infty} \leq 1, i=1, \ldots, l$ such that

$$
x_{i}(k-1)=c_{i}(k-1)+H_{i}(k-1) \theta_{i}(k-1), i=1, \ldots, l .
$$

From the dynamics in (4), in the prediction step, we have that

$$
\begin{aligned}
x_{i}(k)= & \sum_{j \in N_{i}} A_{i j} x_{j}(k-1)+B_{i} u_{i}(k-1)+w_{i}(k-1) \\
= & \sum_{j \in N_{i}} A_{i j}\left(c_{j}(k-1)+H_{j}(k-1) \theta_{j}(k-1)\right) \\
& +B_{i} u_{i}(k-1)+w_{i}(k-1) .
\end{aligned}
$$

From Assumption 1, there exists a vector $\varpi_{i}(k-1)$ with $\left\|\varpi_{i}(k-1)\right\|_{\infty} \leq 1$ for $i=1, \ldots, l$ such that $w_{i}(k-1)=$ $D_{w_{i}} \varpi_{i}(k-1)$. Thus, from (8), we derive

$$
\begin{aligned}
x_{i}(k)= & \sum_{j \in N_{i}} A_{i j}\left(c_{j}(k-1)+H_{j}(k-1) \theta_{j}(k-1)\right) \\
& +B_{i} u_{i}(k-1)+D_{w_{i}} \varpi_{i}(k-1) .
\end{aligned}
$$

Set

$$
\begin{aligned}
\hat{c}_{i}(k) & =\sum_{j \in N_{i}} A_{i j} c_{j}(k-1)+B_{i} u_{i}(k-1), \\
R_{i}(k) & =\left[\operatorname{cat}_{j \in N_{i}}\left\{A_{i j} H_{j}(k-1)\right\}, D_{w_{i}}\right], \\
\eta_{i}(k-1) & =\left[\operatorname{cat}_{j \in N_{i}}\left\{\theta_{j}(k-1)^{T}\right\}, \varpi_{i}(k-1)^{T}\right]^{T},
\end{aligned}
$$

where $\left\|\eta_{i}(k-1)\right\|_{\infty} \leq 1$. Then, we have

$$
x_{i}(k)=\hat{c}_{i}(k)+R_{i}(k) \eta_{i}(k-1) .
$$

From Assumption 1, there exists a vector $\sigma_{i}(k)$ with $\left\|\sigma_{i}(k)\right\|_{\infty} \leq 1$ for $i=1, \ldots, l$ such that $v_{i}(k)=D_{v_{i}} \sigma_{i}(k)$. From the output equation (4), we have

$$
y_{i}(k)-C_{i} x_{i}(k)-D_{v_{i}} \sigma_{i}(k)=0 .
$$

Thus, by replacing $x_{i}(k)$ in (10) with the expression in (9), we obtain

$$
y_{i}(k)-C_{i} \hat{c}_{i}(k)-C_{i} R_{i}(k) \eta_{i}(k-1)-D_{v_{i}} \sigma_{i}(k)=0 .
$$

Pre-multiplying by $\Lambda_{i}$ and the terms of the above equation yields

$$
\begin{aligned}
& \Lambda_{i} y_{i}(k)-\Lambda_{i} C_{i} \hat{c}_{i}(k)-\Lambda_{i} C_{i} R_{i}(k) \eta_{i}(k-1) \\
& \quad-\Lambda_{i} D_{v_{i}} \sigma_{i}(k)=0 .
\end{aligned}
$$

Finally, in the correction step, we add (11) to the right side of (9) obtaining

$$
\begin{aligned}
x_{i}(k)= & \hat{c}_{i}(k)+R_{i}(k) \eta_{i}(k-1)+\Lambda_{i} y_{i}(k) \\
& -\Lambda_{i} C_{i} \hat{c}_{i}(k)-\Lambda_{i} C_{i} R_{i}(k) \eta_{i}(k-1)-\Lambda_{i} D_{v_{i}} \sigma_{i}(k) .
\end{aligned}
$$

By setting $c_{i}(k)$ and $H_{i}(k)$ as in (7), the above equation becomes

$$
x_{i}(k)=c_{i}(k)+H_{i}(k)\left[\begin{array}{c}
\eta_{i}(k-1) \\
-\sigma_{i}(k)
\end{array}\right] .
$$

Since $\left\|\eta_{i}(k-1)\right\|_{\infty} \leq 1$ and $\left\|\sigma_{i}(k)\right\|_{\infty} \leq 1$, we conclude that $x_{i}(k) \in\left\langle c_{i}(k), H_{i}(k)\right\rangle$.

From (6) and (7), we can see that in order to find the distributed state bounding zonotope $\mathcal{X}_{i}(k)$ in an iterative way along the time step $k \in \mathbb{N}$, the correction matrices $\Lambda_{i}$ for $i=1, \ldots, l$ are required. In the following, we will investigate the way to compute $\Lambda_{i}$ for $i=1, \ldots, l$.

\section{B. Computing Correction Matrices}

For state estimation, the objective is to minimize the state estimation errors. Since all estimation errors and uncertainties are propagated and bounded in the distributed zonotope $\mathcal{X}_{i}(k)$, we would like to find $\Lambda_{i}$ for $i=1, \ldots, l$ to minimize the size of these distributed zonotopes. In this work, we use the $P$-radius to measure the size of a zonotope (see Definition 2). In order to guarantee the global stability, we first rewrite the interconnected subsystems (4) as follows.

Denote $x=\left[x_{1}^{T}, \ldots, x_{l}^{T}\right]^{T} \subset \mathbb{R}^{n}$ with $n=\sum_{i=1}^{l} n_{i}$, $u=\left[u_{1}^{T}, \ldots, u_{l}^{T}\right]^{T} \subset \mathbb{R}^{m}$ with $m=\sum_{i=1}^{l} m_{i}$, $y=\left[y_{1}^{T}, \ldots, y_{l}^{T}\right]^{T} \subset \mathbb{R}^{p}$ with $p=\sum_{i=1}^{l} p_{i}, w=$ $\left[w_{1}^{T}, \ldots, w_{l}^{T}\right]^{T} \subset \mathbb{R}^{m_{w}}$ with $m_{w}=\sum_{i=1}^{l} m_{w_{i}}$ and $v=$ $\left[v_{1}^{T}, \ldots, v_{l}^{T}\right]^{T} \subset \mathbb{R}^{m_{v}}$ with $m_{v}=\sum_{i=1}^{l} m_{v_{i}}$. The general system including $l$ agents defined in (4) can be formulated as

$$
\begin{aligned}
x(k+1) & =A x(k)+B u(k)+w(k), \\
y(k) & =C x(k)+v(k),
\end{aligned}
$$

with

$$
A=\left[\begin{array}{ccc}
A_{11} & \cdots & A_{1 l} \\
\vdots & \ddots & \vdots \\
A_{l 1} & \cdots & A_{l l}
\end{array}\right]
$$




$$
B=\operatorname{diag}\left(B_{1}, \ldots, B_{l}\right), C=\operatorname{diag}\left(C_{1}, \ldots, C_{l}\right),
$$

where $w(k) \in\left\langle 0, D_{w}\right\rangle$ and $v(k) \in\left\langle 0, D_{v}\right\rangle, \forall k \in \mathbb{N}$, with $D_{w}=\operatorname{diag}\left(D_{w_{1}}, \ldots, D_{w_{l}}\right)$ and $D_{v}=\operatorname{diag}\left(D_{v_{1}}, \ldots, D_{v_{l}}\right)$.

Proposition 2 (Centralized state bounding zonotope):

Given the the dynamics of the system (12) and suppose that $x(k-1) \in \mathcal{X}(k-1)=\langle c(k-1), H(k-1)\rangle$, for every correction matrix $\Lambda \in \mathbb{R}^{n \times p}$, the following inclusion holds:

$$
x(k) \in \mathcal{X}(k)=\langle c(k), H(k)\rangle,
$$

where

$$
\begin{aligned}
c(k) & =(I-\Lambda C)(A c(k-1)+B u(k-1))+\Lambda y(k), \\
H(k) & =\left[(I-\Lambda C) A H(k-1), \quad(I-\Lambda C) D_{w}, \quad \Lambda D_{v}\right] .
\end{aligned}
$$

Proof: Similar to the proof of Proposition (1), the proof is straightforward and omitted here.

Based on the general state bounding zonotope, we present the conditions of the $P$-radius minimization criterion in the following theorem.

Theorem 1: Given $\mathcal{X}(k)=\langle c(k), H(k)\rangle$ in (13), $\forall k \in$ $\mathbb{N}_{+}$, two scalars $\gamma \in(0,1)$ and $\epsilon>0$. If there exist matrices $P \in \mathbb{S}_{\succ 0}^{n}, Y \in \mathbb{R}^{n \times p}$, diagonal matrices $\Gamma \in \mathbb{S}_{\succeq 0}^{m_{w}}$ and $\Upsilon \in \mathbb{S}_{\succeq 0}^{m_{v}}$ such that

$$
\begin{aligned}
& \operatorname{tr}(\Gamma)+\operatorname{tr}(\Upsilon)<\epsilon, \\
& {\left[\begin{array}{cccc}
\gamma P & \star & \star & \star \\
(P-Y C) A & P & \star & \star \\
0 & D_{w}^{T}(P-Y C)^{T} & \Gamma & \star \\
0 & D_{v}^{T} Y^{T} & 0 & \Upsilon
\end{array}\right] \succ 0,}
\end{aligned}
$$

then it is guaranteed that

$$
V(\mathcal{X}(k)) \leq \gamma V(\mathcal{X}(k-1))+\epsilon, \forall k \in \mathbb{N}_{+},
$$

which leads to $V(\mathcal{X}(\infty)) \leq \frac{\epsilon}{1-\gamma}$ when $k \rightarrow+\infty$.

Proof: From (15), with $P \in \mathbb{S}_{\succ 0}^{n}$ and $\Lambda \in \mathbb{R}^{n \times p}$, for every $H(k-1)$ and $\gamma \in(0,1)$, we have that (15) is equivalent to

$$
\max _{\|\phi\|_{\infty} \leq 1}\|H(k) \phi\|_{P}^{2}-\max _{\|\theta\|_{\infty} \leq 1} \gamma\|H(k-1) \theta\|_{W}^{2}-\epsilon \leq 0 .
$$

Since $\max _{\theta}\|H(k-1) \theta\|_{P}^{2} \geq\|H(k-1) \theta\|_{P}^{2}$ for any $\|\theta\|_{\infty} \leq \stackrel{\theta}{1}$, we obtain the following sufficient condition of (16)

$$
\max _{\|\phi\|_{\infty} \leq 1}\|H(k) \phi\|_{P}^{2}-\gamma\|H(k-1) \theta\|_{W}^{2}-\epsilon \leq 0 .
$$

Let us denote $\phi=\left[\theta^{T}, \varpi^{T}, \sigma^{T}\right]^{T}$ and $Y=R \Lambda$, then

$$
\left[(P-Y C) A, \quad(P-Y C) D_{w}, \quad Y D_{v}\right]=R .
$$

With this notation, we have

$$
H(k) \phi=P^{-1} R\left[(H(k-1) \theta)^{T}, \varpi^{T}, \sigma^{T}\right]^{T} .
$$

Therefore, we rewrite (17) as

$$
\begin{aligned}
& {\left[\begin{array}{c}
H(k-1) \theta \\
\varpi \\
\sigma
\end{array}\right]^{T} R^{T} P^{-1} R\left[\begin{array}{c}
H(k-1) \theta \\
\varpi \\
\sigma
\end{array}\right]} \\
& -\gamma(H(k-1) \theta)^{T} P H(k-1) \theta-\epsilon<0,
\end{aligned}
$$

for any $\|\phi\|_{\infty} \leq 1$. If $\Gamma \in \mathbb{S}_{\succeq 0}^{m_{w}}$ and $\Upsilon \in \mathbb{S}_{\succeq 0}^{m_{v}}$ are diagonal matrices, then we have

$$
\begin{aligned}
& \operatorname{tr}(\Gamma)-\varpi^{T} \Gamma \varpi \geq 0, \forall\|\varpi\|_{\infty} \leq 1, \\
& \operatorname{tr}(\Upsilon)-\sigma^{T} \Upsilon \sigma \geq 0, \quad \forall\|\sigma\|_{\infty} \leq 1 .
\end{aligned}
$$

By adding (20) to (19), we can obtain a sufficient condition

$$
\begin{aligned}
& {\left[\begin{array}{c}
H(k-1) \theta \\
\varpi \\
\sigma
\end{array}\right]^{T} R^{T} P^{-1} R\left[\begin{array}{c}
H(k-1) \theta \\
\varpi \\
\sigma
\end{array}\right]} \\
& -\gamma(H(k-1) \theta)^{T} P H(k-1) \theta-\epsilon \\
& +\operatorname{tr}(\Gamma)-\varpi^{T} \Gamma \varpi+\operatorname{tr}(\Upsilon)-\sigma^{T} \Upsilon \sigma<0 .
\end{aligned}
$$

If (14a) is satisfied, then we obtain

$\left[\begin{array}{c}H(k-1) \theta \\ \varpi \\ \sigma\end{array}\right]^{T}\left(R^{T} P^{-1} R-\left[\begin{array}{ccc}\gamma P & \star & \star \\ 0 & \Gamma & \star \\ 0 & 0 & \Upsilon\end{array}\right]\right)\left[\begin{array}{c}H(k-1) \theta \\ \varpi \\ \sigma\end{array}\right]<0$.

From the above inequality, we have a sufficient condition

$$
R^{T} P^{-1} R-\left[\begin{array}{ccc}
\gamma P & \star & \star \\
0 & \Gamma & \star \\
0 & 0 & \Upsilon
\end{array}\right] \prec 0,
$$

and by changing the sign and applying the Schur complement, we obtain

$$
\left[\begin{array}{cccc}
\gamma P & \star & \star & \star \\
0 & \Gamma & \star & \star \\
0 & 0 & \Upsilon & \star \\
(P-Y C) A & (P-Y C) D_{w} & Y D_{v} & P
\end{array}\right] \succ 0 .
$$

Finally, we obtain (14b) through a linear coordinate transformation by the matrix $T=\left[\begin{array}{llll}I & 0 & 0 & 0 \\ 0 & 0 & I & 0 \\ 0 & 0 & 0 & I \\ 0 & I & 0 & 0\end{array}\right]$ applied to the above inequality.

From the expression of the system (12), it includes $l$ agents in (4). Based on the definition of $A$, we propose the structure of matrices $P$ and $Y$ to be block diagonal matrices in order to find a group of $\Lambda_{i}$ for $i=1, \ldots, l$. Let us define the following structures [18]

$$
\begin{aligned}
& P=\operatorname{diag}\left(P_{1}, \ldots, P_{l}\right), P_{i} \in \mathbb{S}_{\succ 0}^{n_{i}}, i=1, \ldots, l, \\
& Y=\operatorname{diag}\left(Y_{1}, \ldots, Y_{l}\right), Y_{i} \in \mathbb{R}^{n_{i} \times p_{i}}, i=1, \ldots, l .
\end{aligned}
$$

Moreover, $V(\mathcal{X}(\infty)) \leq \frac{\epsilon}{1-\gamma}$ leads to

$$
(x(\infty)-c(\infty))^{T} P(x(\infty)-c(\infty)) \leq \frac{\epsilon}{1-\gamma},
$$

which is an ellipsoid. To minimize the size of this ellipsoid in (22), we can maximize a norm of $P$, as e.g. we choose to maximize $\operatorname{tr}(P)$. Therefore, the correction matrices $\Lambda_{i} \in$ $\mathbb{R}^{n_{i} \times p_{i}}$ for $i=1, \ldots, l$ can be obtained by solving the following optimization problem:

$$
\operatorname{maximize}_{P, L, \Gamma, \Upsilon} \operatorname{tr}(P)
$$

subject to (14).

From the optimal solutions of (23), $\Lambda=P^{-1} Y$ gives $\Lambda=\operatorname{diag}\left(\Lambda_{1}, \ldots, \Lambda_{l}\right)$ with $\Lambda_{i} \in \mathbb{R}^{n_{i} \times p_{i}}$ for $i=1, \ldots, l$. 


\section{Distributed Set-membership Algorithm}

We now summarize the distributed set-membership state estimation approach in the following algorithm.

Distributed set-membership state estimation algorithm.

Offline procedure: Solve the optimization problem (23) with the structured $P$ and $Y$ in (21) to obtain $\Lambda_{i} \in \mathbb{R}^{n_{i} \times p_{i}}$ for $i=1, \ldots, l$.

Online procedure:

- Each agent $i$ sends the state bounding zonotope $\left\langle c_{i}(k-\right.$ 1), $\left.H_{i}(k-1)\right\rangle$ to its neighbors for $i=1, \ldots, l$ and $\forall k \in$ $\mathbb{N}_{+}$;

- Receive the information $\left\langle c_{i}(k-1), H_{i}(k-1)\right\rangle, \forall j \in$ $N_{i}$ from neighbors and obtain the measurement $y_{i}(k)$ and $\left\langle c_{i}(k-1), H_{i}(k-1)\right\rangle$. The distributed zonotope $\left\langle c_{i}(k), H_{i}(k)\right\rangle$ of the agent $i$ is updated by (6) with $\Lambda_{i}$ at time $k$.

- To reduce the order of the zonotope, the overbounding parallelotope of $\left\langle c_{i}(k), H_{i}(k)\right\rangle$ can be obtained using Lemma 1. Denote the over-bounding parallelotope $\left\langle c_{i}(k), H_{i}^{p}(k)\right\rangle$ satisfying $\left\langle c_{i}(k), H_{i}(k)\right\rangle \subseteq$ $\left\langle c_{i}(k), H_{i}^{p}(k)\right\rangle$.

\section{NUMERICAL EXAMPLE}

Given the system including two interconnected subsystems in (4) $(l=2)$ with system matrices:

$$
\begin{aligned}
& A_{11}=\left[\begin{array}{ccc}
0.6848 & -0.0749 & 0.1290 \\
0.6671 & 0.9666 & -0.5852 \\
-0.2789 & -0.1119 & 1.0251
\end{array}\right] \text {, } \\
& A_{12}=\left[\begin{array}{cc}
-0.2488 & -0.0242 \\
-0.9545 & -0.8138 \\
0.3474 & 0.3067
\end{array}\right] \text {, } \\
& A_{21}=\left[\begin{array}{ccc}
-0.2180 & -0.0909 & 0.2027 \\
1.1606 & 0.3804 & -0.9879
\end{array}\right] \text {, } \\
& A_{22}=\left[\begin{array}{cc}
0.8466 & 0.1632 \\
-1.6068 & -0.5130
\end{array}\right] \text {, } \\
& B_{1}=\left[\begin{array}{cc}
0.8 & 0 \\
0 & 0.58 \\
0.6 & 0.8
\end{array}\right], B_{2}=\left[\begin{array}{c}
0.8 \\
-0.75
\end{array}\right] \text {, } \\
& C_{1}=\left[\begin{array}{lll}
1 & 1 & 0 \\
0 & 0 & 1
\end{array}\right], C_{2}=\left[\begin{array}{ll}
1 & 0 \\
0 & 1
\end{array}\right],
\end{aligned}
$$

and $w_{i}(k) \in\left\langle 0, D_{w_{i}}\right\rangle, v_{i}(k) \in\left\langle 0, D_{v_{i}}\right\rangle$ for $i=1,2$ and $\forall k \in \mathbb{N}$, where

$$
\begin{aligned}
& D_{w_{1}}=\left[\begin{array}{ccc}
0.1 & 0 & 0 \\
0 & 0.15 & 0 \\
0 & 0 & 0.25
\end{array}\right], D_{w_{2}}=\left[\begin{array}{cc}
0.1 & 0 \\
0 & 0.15
\end{array}\right], \\
& D_{v_{1}}=\left[\begin{array}{cc}
0.05 & 0 \\
0 & 0.05
\end{array}\right], D_{v_{2}}=\left[\begin{array}{cc}
0.1 & 0 \\
0 & 0.1
\end{array}\right],
\end{aligned}
$$

and the initial state is chosen as $x_{1}(0)=[0.25,1.5,-0.5]^{T} \in$ $\left\langle c_{1}(0), H_{1}(0)\right\rangle$ and $x_{2}(0)=[0.8,0]^{T} \in\left\langle c_{2}(0), H_{2}(0)\right\rangle$, where $c_{1}(0)=x_{1}(0), c_{2}(0)=x_{2}(0)$ and

$$
H_{1}(0)=\left[\begin{array}{ccc}
0.01 & 0 & 0 \\
0 & 0.01 & 0 \\
0 & 0 & 0.01
\end{array}\right], H_{2}(0)=\left[\begin{array}{cc}
0.01 & 0 \\
0 & 0.01
\end{array}\right] \text {. }
$$

The simulations with this numerical example have been carried out in MATLAB and the optimization problems have been solved using the YALMIP [19] with the MOSEK solver [20]. By setting $\gamma=0.8$ and $P$ and $Y$ in block diagonal forms (21), we obtain the optimal correction matrices for the two agents:

$$
\Lambda_{1}=\left[\begin{array}{cc}
0.2433 & 0.1841 \\
0.7567 & -0.1841 \\
0.0375 & 1.1077
\end{array}\right], \Lambda_{2}=\left[\begin{array}{cc}
1.4788 & 0.0093 \\
0.5687 & 1.0129
\end{array}\right] \text {. }
$$

Besides, for a comparison, we also compute the centralized correction matrix $\Lambda^{c}$ with full-dimensional $P$ and $Y$ obtaining

$$
\Lambda^{c}=\left[\begin{array}{cccc}
0.3273 & 0.0877 & 0.1625 & 0.0479 \\
0.6728 & -0.0877 & -0.1625 & -0.0479 \\
0.0920 & 1.0439 & 0.0414 & -0.0247 \\
0.1916 & 0.1993 & 0.6911 & -0.1313 \\
0.2677 & 0.1113 & -0.0047 & 0.7835
\end{array}\right] .
$$

Following the proposed set-membership state estimation algorithm and the the implied centralized algorithm, robust state estimation results are shown in Fig. 1 and Fig. 2. From these plots, we can see that both approaches are able to provide state estimations with generated bounds, and the bounds of the distributed approach are a little larger than the centralized ones. Besides, the optimal values of the optimization problem (23), the mean square error of robust state estimations and a measure of bounds are computed and shown in Table I. With the unstructured $P$, the optimal objective $\operatorname{tr}(P)$ in the centralized approach is a slightly better than the distributed one. As a result, the state estimation error and generated bounds in the centralized approach is a slightly smaller than the distributed ones. However, the distributed approach uses less information and is able to get similar results as the centralized approach.

TABLE I

COMPARISON BETWEEN THE DISTRIBUTED AND CENTRALIZED APPROACHES

\begin{tabular}{lccc}
\hline & $\operatorname{tr}(P)$ & $\operatorname{MSE}$ & $\operatorname{RMS}\left(\sum_{k=0}^{100}(H(k))\right)$ \\
\hline Distributed approach & 799.4855 & 0.0061 & 0.6174 \\
Centralized approach & 799.5274 & 0.0037 & 0.6066 \\
\hline
\end{tabular}

\section{CONCLUSION}

In this paper, we have proposed a distributed setmembership approach based on zonotopes for interconnected systems with coupled states. The interconnected systems are affected by unknown-but-bounded state disturbances and measurement noise. We define a group of parameterized distributed state bounding zonotopes to over-bound uncertain states. For obtaining robust state estimation results, the parameters, that is the correction matrices, are designed by solving the proposed optimization problem based on the $P$ radius minimization. The proposed approach is tested by a numerical example and compared with the centralized approach. 


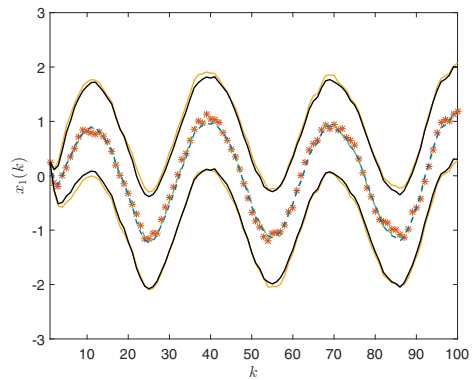

(a) $x_{1}$

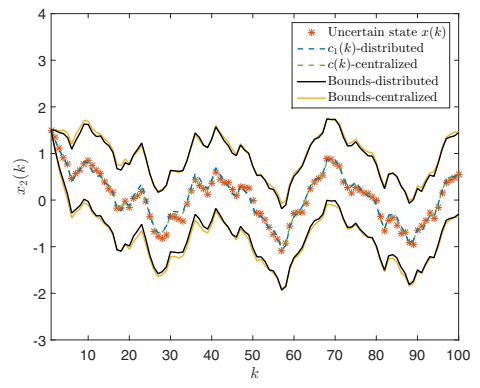

(b) $x_{2}$

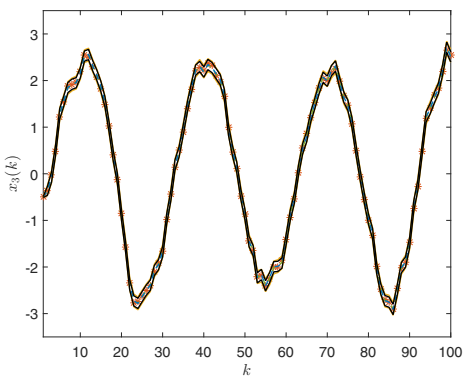

(c) $x_{3}$

Fig. 1. The state estimation result of Agent 1.

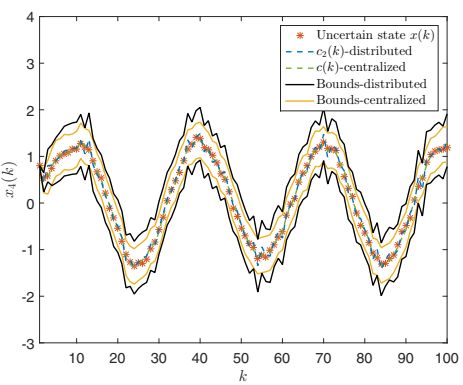

(a) $x_{4}$

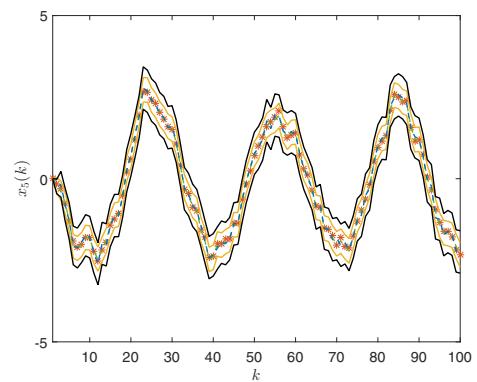

(b) $x_{5}$

Fig. 2. The state estimation result of Agent 2.

\section{ACKNOWLEDGEMENT}

This work has been partially supported by the Spanish State Research Agency (AEI) and the European Regional Development Fund (ERFD) through the project DEOCS (ref. DPI2016-76493) and SCAV (ref. DPI2017-88403-R), the FPI grant (ref. BES-2014-068319) and by AGAUR of Generalitat de Catalunya through the Advanced Control Systems (SAC) group grant (ref. 2017-SGR-482).

\section{REFERENCES}

[1] H. Fawzi, P. Tabuada, and S. Diggavi, "Secure estimation and control for cyber-physical systems under adversarial attacks," IEEE Transactions on Automatic Control, vol. 59, no. 6, pp. 1454-1467, 2014.

[2] Y. Wang, V. Puig, and G. Cembrano, "Non-linear economic model predictive control of water distribution networks," Journal of Process Control, vol. 56, pp. 23-34, 2017.

[3] K. Antoniadou-Plytaria, I. Kouveliotis-Lysikatos, P. Georgilakis, and N. Hatziargyriou, "Distributed and decentralized voltage control of smart distribution networks: Models, methods, and future research," IEEE Transactions on Smart Grid, vol. 8, no. 6, pp. 2999-3008, 2017.

[4] M. Blanke, M. Kinnaert, and M. Lunze, J.and Staroswiecki, "Distributed fault diagnosis and fault-tolerant control," in Diagnosis and Fault-Tolerant Control. Berlin, Heidelberg: Springer, 2016, pp. 467518.

[5] P. Christofides, R. Scattolini, D. Muñoz de la Peña, and J. Liu, "Distributed model predictive control: A tutorial review and future research directions," Computers \& Chemical Engineering, vol. 51, pp. 21-41, 2013.

[6] J. Barreiro-Gomez, N. Quijano, and C. Ocampo-Martinez, "Constrained distributed optimization: A population dynamics approach," Automatica, vol. 69, pp. 101-116, 2016.

[7] X. Yin and J. Liu, "Distributed moving horizon state estimation of two-time-scale nonlinear systems," Automatica, vol. 79, pp. 152-161, 2017.
[8] J. Zeng and J. Liu, "Distributed moving horizon state estimation: Simultaneously handling communication delays and data losses,' Systems \& Control Letters, vol. 75, pp. 56-68, 2015.

[9] V. Puig, P. Cuguero, and J. Quevedo, "Worst-case state estimation and simulation of uncertain discrete-time systems using zonotopes," in European Control Conference, 2001, pp. 1691-1697.

[10] T. Alamo, J. Bravo, and E. Camacho, "Guaranteed state estimation by zonotopes," Automatica, vol. 41, no. 6, pp. 1035-1043, 2005.

[11] T. Alamo, J. Bravo, M. Redondo, and E. Camacho, "A set-membership state estimation algorithm based on DC programming," Automatica, vol. 44, no. 1, pp. 216-224, 2008.

[12] T. Raïssi, D. Efimov, and A. Zolghadri, "Interval state estimation for a class of nonlinear systems," IEEE Transactions on Automatic Control, vol. 57, no. 1, pp. 260-265, 2012.

[13] C. Combastel, "Zonotopes and Kalman observers: Gain optimality under distinct uncertainty paradigms and robust convergence," Automatica, vol. 55, pp. 265-273, 2015.

[14] Y. Wang, V. Puig, and G. Cembrano, "Set-membership approach and Kalman observer based on zonotopes for discrete-time descriptor systems," Automatica, vol. 93, pp. 435-443, 2018.

[15] Y. Wang, T. Alamo, V. Puig, and G. Cembrano, "Distributed zonotopic set-membership state estimation based on optimization methods with partial projection," in 20th IFAC World Congress, 2017, pp. 40394044.

[16] L. Orihuela, S. Roshany-Yamchi, R. García, and P. Millán, "Distributed set-membership observers for interconnected multi-rate systems," Automatica, vol. 85, pp. 221-226, 2017.

[17] V. Le, C. Stoica, T. Alamo, E. Camacho, and D. Dumur, Zonotopes: from guaranteed state-estimation to control. Wiley, 2013.

[18] J. Maestre, D. Muñoz de la Peña, E. Camacho, and T. Alamo, "Distributed model predictive control based on agent negotiation," Journal of Process Control, vol. 21, no. 5, pp. 685-697, 2011.

[19] J. Löfberg, "YALMIP: A toolbox for modeling and optimization in MATLAB," Taipei, Taiwan, 2004. [Online]. Available: http://users.isy.liu.se/johanl/yalmip

[20] MOSEK ApS, The MOSEK optimization toolbox for MATLAB manual. Version 7.1 (Revision 28)., 2015. [Online]. Available: http://docs.mosek.com/7.1/toolbox/index.html 